\title{
Current Tunneling Characterization of Oxidized Black Phosphorus by Graphite Thin Film Electrodes
}

\author{
Received 6 May, 2021; revised 20 May, 2021; accepted 24 May, 2021
}

\author{
Jong Yun Kim ${ }^{a}$, Oh Hun Gwon ${ }^{b}$, Seok-Ju Kang ${ }^{a}$, Hye Ryung Byun ${ }^{a}$, and Young-Jun Yu, \\ a Institute of Quantum Systems, Chungnam National University, Daejeon 34134, Republic of Korea \\ bDepartment of Physics, Chungnam National University, Daejeon 34134, Republic of Korea
}

*Corresponding author E-mail: yjyu@cnu.ac.kr

\begin{abstract}
In this work, oxidized black phosphorus (BP) was trapped in the top and bottom interfaces of graphite thin film electrodes by hexagonal boron nitride (hBN) encapsulation. Upon using partial encapsulation of hBN on BP, the oxidation of bare BP area led to the oxidation of $h B N$ encapsulated whole BP, and this oxidized BP could be confined in the hBN layer. Furthermore, by attaching graphite thin film electrodes on and underneath the oxidized BP layer, charge carrier injection and extraction behavior from measuring the current tunneling was characterized by applying a bias voltage between the top and the bottom graphite thin film electrodes. The electrical characteristics according to applied bias voltage was confirmed with a double log plot. It was found that the ohmic current region exists in the low voltage state, and the space-chargelimited conduction region exists in the high voltage state.
\end{abstract}

Keywords: Black phosphorus, Hexagonal boron nitride, Graphite thin film, Oxidation

\section{Introduction}

Black phosphorus (BP), with its high charge carrier mobility $(>\sim$ $\left.200 \mathrm{~cm}^{2} / \mathrm{Vs}\right)$ and resulting bandgap $(0.3-2.0 \mathrm{eV})$, has been favored in atomically thin and high-speed semiconductor devices [1-8]. However, there are certain challenges with applying BP in optical and electrical devices, with the instability (i.e., oxidation) of BP in air being a critical one. Since the oxidation period of BP in air in both singleand multi-layers is reported to be from 1 hour to 2 weeks, oxidation on a thick BP surface and the disappearance of thin BP can be easily observed by optical microscopy [9-12]. Because of the instability of BP in air, various methods to protect BP from oxygen have been proposed. Although hexagonal boron nitride $(\mathrm{hBN})$ has been widely researched regarding its protective capability of $\mathrm{BP}[13,14]$, BP without full hBN encapsulation still leads to oxidation, which is initiated in the area exposed to air. It is difficult to avoid BP oxidation completely. However, if we change our point of view oppositively, an artificial oxidized layer is able to be generated in the interface between two-dimensional layers. In this work, upon preparing partially encapsulated $\mathrm{BP}$ by $\mathrm{hBN}$, an oxidized $\mathrm{BP}$ layer under $\mathrm{hBN}$ by initially oxidizing from bare BP was created. The current tunneling characterization of this oxidized BP layer was investigated by using graphene-electrodes sandwiched heterostructures. Because oxides can be applied to memory devices [15-19], the electrical characterization of an oxidized BP layer will make a good reference for atomically thin memory device applications.

\section{Experimental details}

In order to study the current transport behavior of oxidized BP, we employed graphite thin film electrodes, as shown in Fig. 1. The

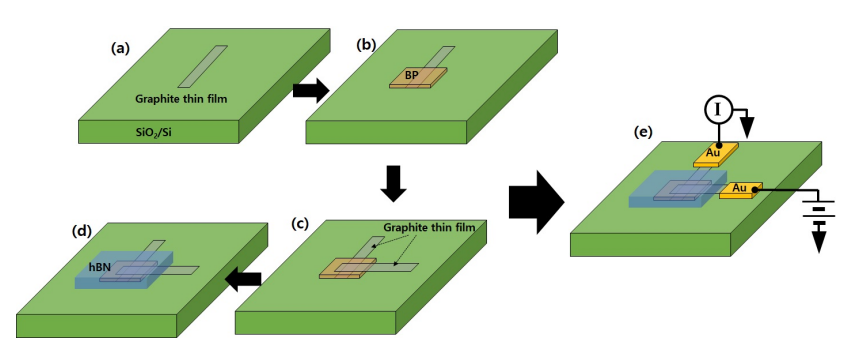

Figure 1. Schematic diagram of $h B N / T G / B P / B G$ heterostructure fabrication. (a) Mechanical exfoliation of $\mathrm{BG}$ on $\mathrm{SiO}_{2} / \mathrm{Si}$ substrate. (b) Transfer of BP to $\mathrm{BG} / \mathrm{SiO}_{2} / \mathrm{Si}$ substrate. (c) Transfer of $\mathrm{TG}$ to $\mathrm{BP} / \mathrm{BG} / \mathrm{SiO}_{2} / \mathrm{Si}$ heterostructure. (d) Encapsulation of $\mathrm{hBN}$ on $\mathrm{TG} / \mathrm{BP} / \mathrm{BG} / \mathrm{SiO}_{2} / \mathrm{Si}$ heterostructure. (e) $\mathrm{Cr} / \mathrm{Au}$ electrodes contact with TG and BG and electrical characterization.

bottom graphite thin film (BG) on 280 -nm-thick $\mathrm{SiO}_{2}$ substrate was mechanically exfoliated [Fig. 1(a)] and the BP flake was transferred to the BG [Fig. 1(b)]. The top graphite thin film (TG) was stacked on the $\mathrm{BP} / \mathrm{BG} / \mathrm{SiO}_{2}$ area [Fig. 1(c)] for measuring the current tunneling behavior; this $\mathrm{TG} / \mathrm{BP} / \mathrm{BG} / \mathrm{SiO}_{2}$ heterostructure was encapsulated by $\mathrm{hBN}$, as shown in Fig. 1(d). This two-dimensional material stacking process was performed using a micro-transferring system with polydimethylsiloxane (PDMS) stamping $[14,19,20]$. The TG and the BG were then contacted using $\mathrm{Cr} / \mathrm{Au}(10 \mathrm{~nm} / 100 \mathrm{~nm})$ electrodes via normal e-beam lithography [see Fig. 1(e)]. Upon employing a semiconductor parameter analyzer (Keithley 4200), the charge carrier transport behavior of oxidized BP between the TG and the BG was characterized, as shown in Fig. 1(e).

This is an Open Access article distributed under the terms of the Creative Commons Attribution Non-Commercial License (http://creativecommons.org/licenses/by-nc/3.0) which permits unrestricted non-commercial use, distribution, and reproduction in any medium, provided the original work is properly cited. 
(a)
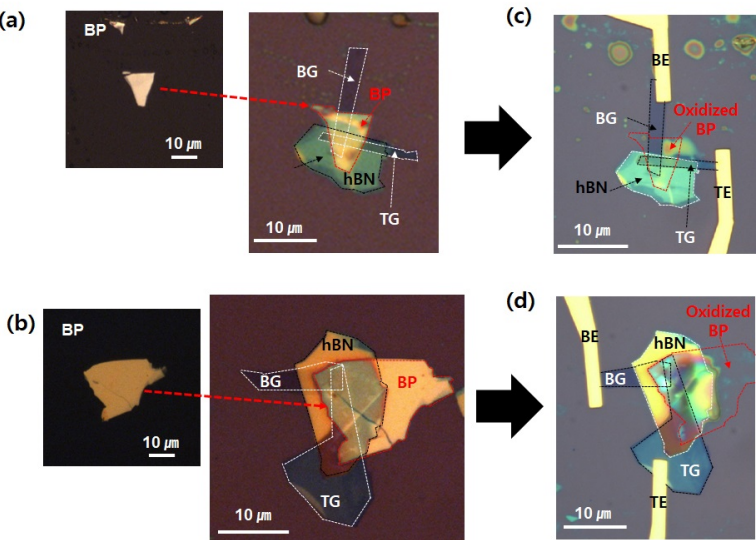

(d)

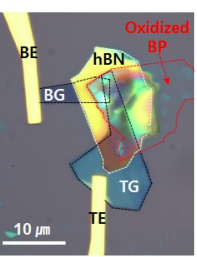

Figure 2. (a) Optical image of (left) BP layer ( $40 \mathrm{~nm}$ thickness) on PDMS trans ferring polymer layer, and (right) hBN/TG/BP/BG/SiO $/$ Si heterostructure with $10 \mathrm{~nm}$ thick hBN. (b) Optical image of (left) BP layer ( $60 \mathrm{~nm}$ thickness) on PDMS transferring polymer layer, and (right) hBN/TG/BP/BG/SiO $/$ Si heterostructure with $90 \mathrm{~nm}$ thick hBN. Thicknesses of TG and BG are around 10 and $5 \mathrm{~nm}$, respectively. (c) Optical image of hBN/TG/BP/BG/SiO $/ 2$ Si heterostructure ( 10$\mathrm{nm}$-thick hBN and 40-nm-thick BP) after contact with Au top-electrode (TE) and bottom-electrode (BE) on TG and BG, respectively. (d) Optical image of $\mathrm{hBN} / \mathrm{TG} / \mathrm{BP} / \mathrm{BG} / \mathrm{SiO}_{2} / \mathrm{Si}$ heterostructure $(\sim 90-\mathrm{nm}$-thick hBN and $~ 60$-nm-thick $\mathrm{BP}$ ) after contact with $\mathrm{Au} T \mathrm{TE}$ and $\mathrm{BE}$ on $\mathrm{TG}$ and $\mathrm{BG}$, respectively. The oxidized $\mathrm{BP}$ disappears due to exposure to air during the Au electrode contact fabrication process.

\section{Results and discussion}

Figure 2 shows the hBN/TG/BP/BG heterostructure with a thickness of $\sim 5$ and $\sim 10 \mathrm{~nm}$ for the $\mathrm{BG}$ and TG, respectively. The hBN and BP had different thicknesses: thin $\mathrm{hBN}(\sim 10 \mathrm{~nm})$, BP $(\sim 40 \mathrm{~nm})$ for Fig. 2(a); and thick hBN ( $90 \mathrm{~nm})$, BP $(\sim 60 \mathrm{~nm})$ for Fig. 2(b). While the BP layers in Figs. 2(a) and 2(b) can be clearly observed by optical microscopy when the process of stacking was just completed for the $\mathrm{hBN} / \mathrm{TG} / \mathrm{BP} / \mathrm{BG}$ heterostructures, the disappearing BP layers in the heterostructures appeared during the Au electrode contact fabrication process [see Figs. 2(c) and 2(d)]. This behavior is well known
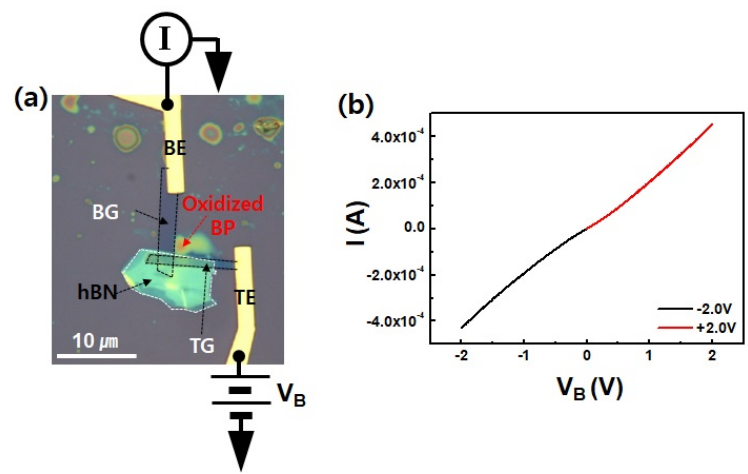

Figure 3. (a) Optical image of hBN/TG/BP/BG/SiO $/$ Si heterostructure ( 10-nmthick hBN and 40-nm-thick BP) after contact with Au electrodes on TG and BG. Schematic diagram of tunneling current from applying a bias voltage between TG and BG electrodes. (b) Current (I) as a function of bias voltage $\left(V_{B}\right)$ between TG $(\mathrm{TE})$ and $\mathrm{BG}(\mathrm{BE})$ electrodes. The red and black lines represent forward $(+)$ and backward (-) sweep.

as the oxidation of BP in air [9-12]. A period of $\sim 1$ week was given for the Au electrode contact fabrication on the TG and BG. This was a sufficient length of time to see the BP layer disappear due to oxidation in air.

According to this phenomenon, thin $\mathrm{BP}(\sim 40 \mathrm{~nm})$ encapsulated by thin $\mathrm{hBN}(\sim 10 \mathrm{~nm})$ in $\mathrm{hBN} / \mathrm{TG} / \mathrm{BP} / \mathrm{BG}$ heterostructures exhibits an almost linear current curve $(\mathrm{I})$ as a function of the bias voltage $\left(\mathrm{V}_{\mathrm{B}}\right)$ between TG and BG, as shown in Fig. 3. As confirmation of the oxidized $\mathrm{BP}$ in the hBN/TG/BP/BG heterostructures in Fig. 3(a), we did not observe even oxidized BP layer in overlapped by TG and BG area. The $\mathrm{I}-\mathrm{V}_{\mathrm{B}}$ curve for the $\mathrm{hBN} / \mathrm{TG} / \mathrm{BP} / \mathrm{BG}$ heterostructure exhibited a tunnel resistance of $\sim 5 \mathrm{k} \Omega$, and this value could be corresponded to only contact resistance between TG and BG electrodes without a tunnel barrier [21].

Meanwhile, for thick BP $(\sim 60 \mathrm{~nm})$ encapsulated by thick hBN ( $90 \mathrm{~nm}$ ) in the $\mathrm{hBN} / \mathrm{TG} / \mathrm{BP} / \mathrm{BG}$ heterostructure, slightly bright areas [marked by the red dashed boxes and arrows in Fig. 4(a)] were observed in the overlapping TG and BG area. The hysteresis of the cur-
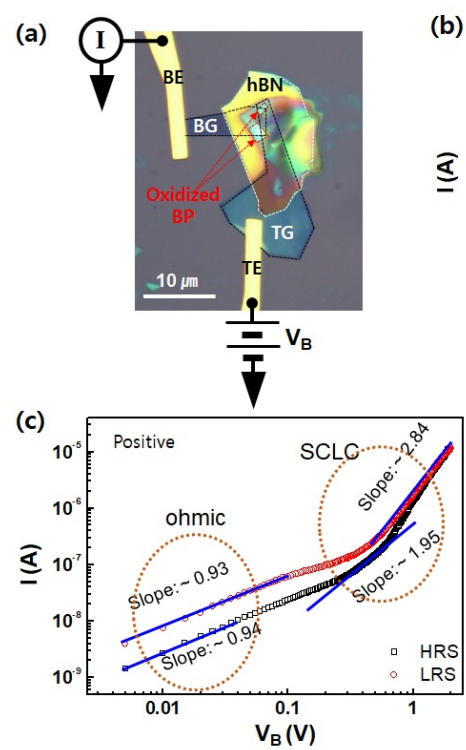
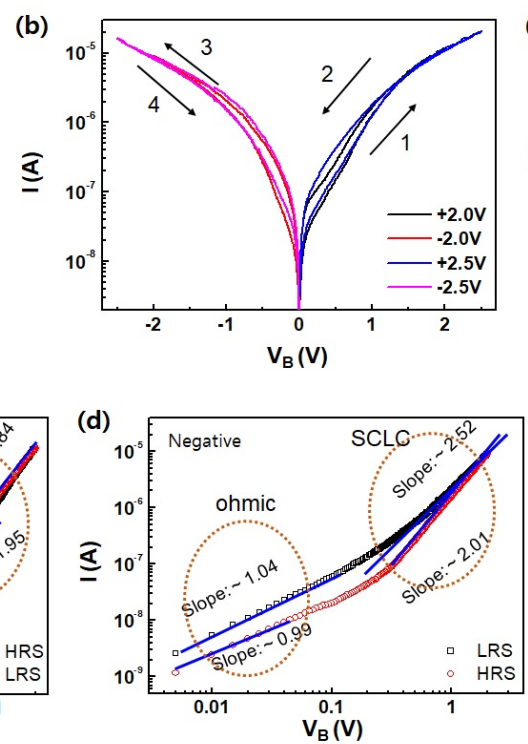
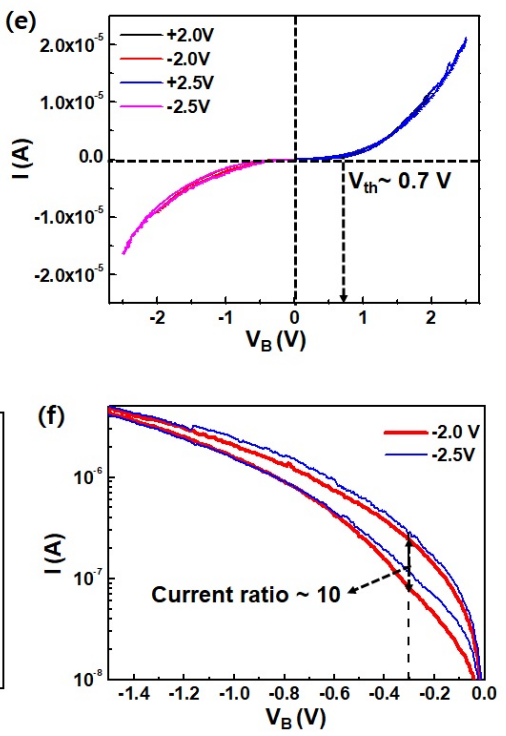

Figure 4. (a) Optical image of hBN/TG/BP/BG/SiO $/$ Si heterostructure ( 90-nm-thick hBN and 60-nm-thick BP) after contact with Au electrodes on TG and BG. Schematic diagram of tunneling current from applying bias voltage between TG and BG electrodes. (b) Current (I) as a function of bias voltage ( $\left.V_{B}\right)$ between TG (TE) and BG (BE) electrodes. The double logarithmic scale plots of (c) positive and (d) negative voltage sweep region for (b) I- $V_{B}$ curve. (e) Linear scale (I) as a function of $V_{B}$ between $T G(T E)$ and $B G(B E)$ electrodes. (f) Enlarged I- $V_{B}$ curve of (b). The red and blue lines represent -2.0 and $-2.5 \mathrm{~V}$ dual sweep, respectively. 
rent curve could be seen for the forward and backward sweep of $V_{B}$ [marked by arrows and numbers 1-4 in Fig. 4(b)]. By employing a logarithmic scale of both $I$ and $V_{B}$ values for positive and negative voltage sweep regions, as shown in Figs. 4(c) and 4(d), respectively, the ohmic and space-charge-limited conductance regions with $\mathrm{I}-\mathrm{V}_{\mathrm{B}}$ curve slops as $\sim 1$ and $\sim 2$, respectively, could be characterized [22-24]. This indicates that the potential barrier of oxidized BP resulting in ohmic behavior under low $\mathrm{V}_{\mathrm{B}} \sim 0.1 \mathrm{~V}$ is suppressed by charge carrier injection on oxidized BP, leading to space-charge-limited conductance behavior for positive and negative $V_{B}$ sweep larger than $\pm 0.1 \mathrm{~V}$, respectively. As a result, the $I-V_{B}$ exhibited a general tunneling behavior with a threshold voltage $\left(\mathrm{V}_{\mathrm{th}}\right)$ of $\sim \pm 0.6 \mathrm{~V}$, as shown in Fig. $4(\mathrm{e})$. This indicates that there is oxidized BP between the TG and the BG electrodes, and the storing and releasing charge carrier on oxidized BP between TG and BG for the forward and backward sweep of $V_{B}$ gave rise to a current ratio of 10 for the hysteresis area at $V_{B}=-0.3 \mathrm{~V}$, as shown in Fig. 4(f). Thus, we confirmed that the oxidized BP was confined in the heterojunction with thick $\mathrm{hBN}(\geq 90 \mathrm{~nm})$ encapsulation. As a result, although the current ratio of $\sim 10$ was not particularly large, it nonetheless suggested the potential for oxidized BP layer utilization for memory application.

\section{Conclusions}

In this work, an oxidized black phosphorus (BP) layer in the interface between graphite thin film electrodes with hexagonal boron nitride (hBN) encapsulation was examined. For thick hBN (>90 nm) encapsulation, the oxidized BP layer can be locked in the hBN layer, with the charge carrier storing or releasing on this oxide layer with a forward (+) and backward (-) sweep of a bias voltage between the top and bottom graphite thin film electrodes. Although the current ratio and memory window is not especially large, the potential for oxidized BP layer application in memory devices remains meaningful.

\section{Acknowledgements}

This work was supported by the research fund of Chungnam National University.

\section{References}

[1] L. Li, Y. Yu, G. Ye, Q. Ge, X. Ou, H. Wu, D. Feng, X. Chen, and Y. Zhang, Nat. Nanotechnol. 9, 372 (2014).

[2] D. K. Kim, S. B. Hong, K. Jeong, C. Lee, H. Kim, and M. H. Cho, ACS Nano 13, 1683 (2019).

[3] D. Lee, Y. Choi, E. Hwang, M. S. Kang, S. Lee, and J. H. Cho, Nanoscale 8, 9107 (2016).

[4] J. Liu, Y. Chen, Y. Li, H. Zhang, S. Zheng, and S. Xu, Photonics Res. 6, 198 (2018).

[5] T. Chen, P. Zhao, X. Guo, and S. Zhang, Nano Lett. 17, 2299 (2017).

[6] M. Zhu, S. Kim, L. Mao, M. Fujitsuka, J. Zhang, X. Wang, and T. Majima, J. Am. Chem. Soc. 139, 13234 (2017).

[7] S. Yan, B. Wang, Z. Wang, D. Hu, X. Xu, J. Wang, and Y. Shi, Biosens. Bioelectron. 80, 34 (2016).

[8] J. Zhou, Z. Li, M. Ying, M. Liu, X. Wang, X. Wang, L. Cao, H. Zhang, and G. Xu, Nanoscale 10, 5060 (2018).

[9] Y. Huang et al., Preprint at https://arxiv.org/ftp/arxiv/papers/ 1511/1511.09201.pdf (2015).

[10] J. S. Kim, Y. Liu, W. Zhu, S. Kim, D. Wu, L. Tao, A. Dodabalapur, K. Lai, and D. Akinwande, Sci. Rep. 5, 8989 (2015).

[11] B. Yang et al., Adv. Mater. 28, 9408 (2016).

[12] J. Kim, S. K. Baek, K. S. Kim, Y. J. Chang, and E. J. Choi, Curr. Appl. Phys. 16, 165 (2016).

[13] R. A. Doganov et al., Nat. Commun. 6, 6647 (2015).
[14] Z. H. Wang, A. Islam, R. Yang, X. Q. Zheng, and P. X. L. Feng, J. Vac. Sci. Technol. B 33, 052202 (2015).

[15] M. H. Jeong, D. H. Kwak, H. S. Ra, A. Y. Lee, and J. S. Lee, ACS Appl. Mater. Interfaces 10, 19069 (2018).

[16] D. Kumar, R. Aluguri, U. Chand, and T. Y. Tseng, Ceram. Int. 43, S547 (2017)

[17] Y. Yang, S. Choi, and W. Liu Nano Lett. 13, 2908 (2013).

[18] M. Chen, H. Nam, S. Mi, G. Priessnitz, I. M. Gunawan, and X. Liang, ACS Nano 8, 4023 (2014).

[19] H. Shen, J. Ren, J. Li, Y. Chen, S. Lan, J. Wang, H. Wang, and D. Li, ACS Appl. Mater. Inter. 12, 58428 (2020).

[20] C. Hou, L. Yang, B. Li, Q. Zhang, Y. Li, Q. Yue, Y. Wang, Z. Yang, and L. Dong, Sensors 18, 1668 (2018).

[21] G. H. Lee, Y. J. Yu, C. Lee, C. Dean, K. L. Shepard, P. Kim, and J. Hone, Appl. Phys. Lett. 99, 243114 (2011).

[22] H. Y. Jeong et al., Nano Lett. 10, 4381 (2010).

[23] A. Rose, Phys. Rev. 97, 1538 (1955).

[24] R. Dong et al., Appl. Phys. Lett. 90, 042107 (2007). 